\title{
Effect of Cumulative Dexamethasone Dose during Concomitant Chemoradiation on Lymphopenia in Patients with Newly Diagnosed Glioblastoma
}

\author{
Changik Lee ${ }^{1}$ (D), Stephen Ahn ${ }^{1}$ (D), Jae-Sung Park', Jin Ho Song ${ }^{2}$, Yong-Kil Hong ${ }^{1}$, Sin-Soo Jeun ${ }^{1}$ \\ Departments of ${ }^{1}$ Neurosurgery, ${ }^{2}$ Radiation Oncology, Seoul St. Mary's Hospital, College of Medicine, The Catholic University of Korea, Seoul, Korea
}

Received March 2, 2020

Revised March 30, 2020

Accepted April 7, 2020

\section{Correspondence}

Stephen Ahn

Department of Neurosurgery,

Seoul St. Mary's Hospital,

College of Medicine,

The Catholic University of Korea,

222 Banpodae-ro, Seocho-gu,

Seoul 06591, Korea

Tel: $+82-2-2258-7535$

Fax: +82-2-2258-1853

E-mail: purestp@gmail.com
Background Lymphopenia frequently occurs after concomitant chemoradiation (CCRT) in patients with glioblastoma (GBM) and is associated with worse overall survival (OS). A few studies have tried to identify risk factors for lymphopenia; however, the results were not clear. We aimed to identify potential risk factors for lymphopenia, focusing on the use of dexamethasone to control cerebral edema in patients with GBM.

Methods The electronic medical records of 186 patients with newly diagnosed GBM treated at our institution between 2009 and 2017 were retrospectively examined. Acute lymphopenia was defined as total lymphocyte count less than 1,000 cells/ $\mu \mathrm{L}$ at 4 weeks after completion of CCRT. Multivariate logistic regression analysis was used to identify independent risk factors for lymphopenia, and Cox regression analysis was used to identify independent risk factors for OS.

Results Of the 125 eligible patients, 40 patients (32.0\%) developed acute lymphopenia. Female sex and median daily dexamethasone dose $\geq 2 \mathrm{mg}$ after initiation of CCRT were independent risk factors for acute lymphopenia on multivariate analysis. Acute lymphopenia, extent of surgical resection, and performance status were associated with OS; however, dexamethasone use itself was not an independent risk factor for poor OS.

Conclusion Female sex, median daily dexamethasone dose $\geq 2 \mathrm{mg}$ after initiation of CCRT until 4 weeks after completion of CCRT may be associated with acute lymphopenia. However, dexamethasone use itself did not affect OS in patients newly diagnosed with GBM. These results should be validated by further prospective studies controlling for other confounding factors.

Key Words ～Glioblastoma; Lymphopenia; Lymphocyte count; Dexamethasone; Glucocorticoid; Chemoradiotherapy.

\section{INTRODUCTION}

Lymphopenia, defined as a reduction in total lymphocyte count (TLC), frequently occurs after concomitant chemoradiation (CCRT) for glioblastoma (GBM), and it was recently suggested as a novel biomarker associated with overall survival (OS) in newly diagnosed GBM [1-6]. As lymphocytes play a major role in the immune response to eliminate malig-

This is an Open Access article distributed under the terms of the Creative Commons Attribution Non-Commercial License (https://creativecommons.org/licenses/by-nc/4.0) which permits unrestricted non-commercial use, distribution, and reproduction in any medium, provided the original work is properly cited.

Copyright $\odot 2020$ The Korean Brain Tumor Society, The Korean Society for NeuroOncology, and The Korean Society for Pediatric Neuro-Oncology nant cells, an insufficient number of circulating lymphocytes could weaken the immune system's control of GBM progression [7-10].

Several clinical factors have been suggested as contributors to lymphopenia including sex, age, irradiated brain volume, chemotherapy, and the use of dexamethasone [11-18]. Radiotherapy (RT) was found to be significantly associated with lymphopenia in GBM patients $[10,11,14-16,18,19]$. Dexamethasone, which is a commonly prescribed drug for controlling brain edema and improving performance of GBM patients, is also known for its potent immunosuppressive effects [20-22]. However, its effect on inducing lymphopenia in GBM patients remains controversial $[11,14,15,17]$. Three previous studies 
did not find an association between the use of dexamethasone and acute lymphopenia $[11,14,15]$, but another study showed that increased use of dexamethasone during CCRT was an independent risk factor for acute lymphopenia after CCRT [17]. As numerous immunotherapeutic agents are being developed for GBM, it is critical to minimize iatrogenic lymphopenia to maintain sufficient immunity to fight against cancer cells $[10,23]$.

In this context, we attempted to elucidate the clinical factors associated with lymphopenia following CCRT in patients with newly diagnosed GBM focusing specifically on the use of dexamethasone. We examined the overall cumulative dose of dexamethasone from initiation of CCRT to 4 weeks after completion of CCRT to facilitate a more accurate evaluation of this association.

\section{MATERIALS AND METHODS}

\section{Patient selection}

Our study was approved by the Institutional Review Board of Seoul St. Mary's Hospital (KC19RESI0168). The need for informed consent was waived due to the retrospective nature of our study. The electronic medical records (EMR) of $186 \mathrm{pa}-$ tients with newly diagnosed GBM who were treated at our institution between August 2009 and December 2017 were retrospectively examined. The eligibility criteria were as follows: 1) newly diagnosed GBM confirmed by craniotomy or stereotactic biopsy, 2) completion of CCRT at our institution, 3) accessible baseline and follow-up complete blood count (CBC). The exclusion criteria were as follows: 1) prior history of diagnosed malignancies, chemotherapy, or radiation due to other medical illnesses, 2) evidence of acute infection or autoimmune disease at the time of blood sampling.

\section{Clinical variables and treatment protocols}

Patient characteristics included sex, age, performance status, extent of resection (EOR), pathologic findings, radiation dosage and fraction, radiation volume, radiation modalities, dose of dexamethasone used, and CBC. Performance status at baseline was measured using the Eastern Cooperative Oncology Group (ECOG) performance score. EOR was measured by comparing radiologic findings on MRI at baseline and within 48 hours after surgery. Resection of $90 \%$ or more of the tumor volume was considered gross total resection (GTR) and $<90 \%$ was considered non-GTR [24]. Isocitrate dehydrogenase 1 (IDH1) mutation was evaluated by immunohistochemistry or direct sequencing.

$\mathrm{O}^{6}$-methylguanine-DNA methyltransferase (MGMT) gene methylation status was evaluated by polymerase chain reaction.

We used navigation and intraoperative monitoring in all surgeries and we also performed awake surgery or 5-aminolevulinic acid fluorescence-guided surgery, for achieving maximal safe resection, when indicated. CCRT was started within 28 days of surgical resection if possible, considering wound healing, the patient's condition, and RT schedules [25]. Radiation was administered at a dosage of either 5,940 cGy for 33 fractions or $6,000 \mathrm{cGy}$ for 30 fractions, regardless of age. RT was conducted with either three-dimensional conformal RT (3DRT) or intensity-modulated RT (IMRT). Temozolomide (TMZ) dosages were $75 \mathrm{mg} / \mathrm{m}^{2}$ during CCRT and $150-200 \mathrm{mg} / \mathrm{m}^{2}$ for adjuvant therapy. We used dexamethasone, considering the patient's neurologic status, general condition, and radiologic findings after surgery to control brain edema and improve patient performance. Other steroids including prednisolone were not prescribed.

\section{TLC and dexamethasone}

We collected results of blood tests, including white blood cell count and the composition of neutrophils and lymphocytes, at baseline and 4 weeks after completion of CCRT. TLC were calculated from these data for the analysis.

We also reviewed prescribed dexamethasone doses using EMR from initiation of CCRT until 4 weeks after the completion of CCRT, which was the same day blood samples were collected for calculating TLC. We estimated the cumulative dexamethasone dose from the EMR. Median dexamethasone dose prescribed during this period was calculated by dividing the cumulative dexamethasone dose $(\mathrm{mg})$ by the days from initiation of CCRT to 4 weeks after the completion of CCRT. For example, if a patient took a cumulative dexamethasone dose of $200 \mathrm{mg}$ over 80 days from initiation of CCRT to 4 weeks after completion of, the median daily dexamethasone dose was $2.5 \mathrm{mg}$. In our study, the median period from initiation of CCRT until 4 weeks after completion of CCRT was 78.3 (range 65-98) days.

\section{Statistical analysis}

Lymphopenia was defined as TLC $<1,000$ cells $/ \mu \mathrm{L}$ at 4 weeks after completion of CCRT, based on previous reports. The primary endpoint in our study was OS. OS was calculated using the time interval from date of initial surgery to death. Dates of death were obtained from the Korea Central Cancer Registry database. Patients who were confirmed alive on July 30, 2019 were censored. The median follow-up period was 26.1 (range 4-117) months.

All clinical variables were characterized with descriptive statistics. Differences between groups in which dexamethasone was and was not used were compared with Fisher's exact test or the chi-squared test. A test of normality was performed for continuous variables. Univariate and multivariate analyses 
were conducted using the Cox proportional regression model. The hazard ratio (HR) and 95\% confidence intervals (CIs) were calculated. Multivariate analysis was performed using the variables with $p$-values $<0.2$, and $p$-values $<0.05$ were considered statistically significant. Statistical analysis was performed using R Statistical Software (Version 3.4.4; R Foundation for Statistical Computing, Vienna, Austria).

\section{RESULTS}

\section{Patient characteristics}

We included 125 patients who met our eligibility criteria. Of them, 89 patients who did not take any dexamethasone were assigned to the "not used group" and 36 patients who took dexamethasone were assigned to the "used group." Baseline patient characteristics are shown in Table 1. The "used group" had poor ECOG performance score at baseline and had more patients who underwent non-GTR, while other clinical variables including sex, age, IDH1 mutation status, presence of MGMT promoter methylation, median planning treatment volume (PTV), median TLC at baseline and at 4 weeks after completion of CCRT, and proportion of lymphopenia were not significantly different. The median daily dexamethasone dose $(\mathrm{mg})$ in the "used group" was 1.9 (range 0.1$8.3) \mathrm{mg} /$ day. Eighteen of 36 patients (50\%) took a median dexamethasone dose less than $2.0 \mathrm{mg} /$ day, 12 patients (33.3\%) from 2.0 to $4.0 \mathrm{mg} / \mathrm{day}$, and 6 patients (16.6\%) more than 4 $\mathrm{mg} /$ day.

Table 1. Characteristics of patients with newly diagnosed glioblastoma who completed CCRT according to dexamethasone use

\begin{tabular}{|c|c|c|c|c|}
\hline Variables & $\begin{array}{l}\text { Dexamethasone } \\
\text { not used }(n=89)\end{array}$ & $\begin{array}{c}\text { Dexamethasone } \\
\text { used }(n=36)\end{array}$ & Total $(n=125)$ & $p$-value \\
\hline Sex & & & & 0.268 \\
\hline Female & $38(42.7)$ & $20(55.6)$ & $58(46.4)$ & \\
\hline Male & $51(57.3)$ & $16(44.4)$ & $67(53.6)$ & \\
\hline Age at diagnosis (yr) & $59.0(20-79)$ & $59.0(30-79)$ & $59.0(20-79)$ & 0.667 \\
\hline Age $\geq 65 \mathrm{yr}$ & $29(32.6)$ & $9(25.0)$ & $38(30.4)$ & 0.535 \\
\hline ECOG at baseline & & & & 0.053 \\
\hline $0-1$ & $59(66.3)$ & $17(47.2)$ & $76(60.8)$ & \\
\hline $2-3$ & $30(33.7)$ & $19(52.8)$ & $49(38.2)$ & \\
\hline Extent of resection & & & & $<0.001$ \\
\hline GTR & $70(78.7)$ & $15(41.7)$ & $85(68.0)$ & \\
\hline Non-GTR & $19(21.3)$ & $21(58.3)$ & $40(32.0)$ & \\
\hline IDH1 mutation & & & & 0.252 \\
\hline Yes & $5(5.6)$ & $0(0.0)$ & $5(4.0)$ & \\
\hline No & $40(44.9)$ & $20(55.6)$ & $60(48.0)$ & \\
\hline Unknown & $44(49.4)$ & $16(44.4)$ & $60(48.0)$ & \\
\hline MGMT methylation & & & & 0.391 \\
\hline Yes & $35(39.3)$ & $15(41.7)$ & $50(40.0)$ & \\
\hline No & $35(39.3)$ & $4(11.1)$ & $52(41.6)$ & \\
\hline Unknown & $19(21.3)$ & $17(47.2)$ & $23(18.4)$ & \\
\hline RT technique & & & & 0.352 \\
\hline IMRT & $23(25.8)$ & $13(36.1)$ & $36(28.8)$ & \\
\hline 3DRT & $66(74.2)$ & $23(63.9)$ & $89(71.2)$ & \\
\hline PTV $\left(\mathrm{cm}^{3}\right)$ & $210.3(14.7-691.4)$ & $280.7(12.9-568.4)$ & $214.1(12.9-691.4)$ & 0.541 \\
\hline Daily dexamethasone dose (mg) & $0.0(0.0-0.0)$ & $1.9(0.1-8.3)$ & - & \\
\hline$<2 \mathrm{mg} /$ day & - & $18(50.0)$ & - & \\
\hline $2-4 \mathrm{mg} /$ day & - & $12(33.3)$ & - & \\
\hline$>4 \mathrm{mg} /$ day & - & $6(16.7)$ & - & \\
\hline TLC at baseline $($ cells $/ \mu \mathrm{L})$ & $1,601(256-4,950)$ & $1,569(548-4,410)$ & $1,583(256-4,950)$ & 0.883 \\
\hline TLC 4 weeks after completion of CCRT (cells/ $\mu \mathrm{L})$ & $1,330(471-4,632)$ & $1,078(240-2,187)$ & $1,300(240-4,632)$ & 0.113 \\
\hline TLC $<1,000$ cells $/ \mu \mathrm{L} 4$ weeks after completion of CCRT & $24(27.0)$ & $16(44.4)$ & $40(32.0)$ & 0.092 \\
\hline
\end{tabular}

Data are presented as number (\%) or median (range). CCRT, concomitant chemoradiation; ECOG, Eastern Cooperative Oncology Group; GTR, gross total resection; IDH, isocitrate dehydrogenase; IMRT, intensity-modulated radiation therapy; 3DRT, three-dimensional conformal radiation therapy; PTV, planning treatment volume; TLC, total lymphocyte count 
Table 2. Univariate and multivariate Cox regression for overall survival

\begin{tabular}{|c|c|c|c|c|}
\hline \multirow{2}{*}{ Parameters } & \multicolumn{2}{|c|}{ Univariate analysis } & \multicolumn{2}{|c|}{ Multivariate analysis* } \\
\hline & Hazard ratio $(95 \% \mathrm{CI})$ & $p$-value & Hazard ratio $(95 \% \mathrm{CI})$ & $p$-value \\
\hline Sex (male vs. female) & $0.74(0.48-1.14)$ & 0.177 & $0.99(0.62-1.61)$ & 0.997 \\
\hline Age $\geq 65$ yr (vs. $<65$ yr) & $1.16(0.73-1.87)$ & 0.525 & - & - \\
\hline ECOG 0 or 1 (vs. 2 or 3$)$ & $0.41(0.26-0.64)$ & $<0.001$ & $0.46(0.28-0.76)$ & 0.002 \\
\hline Incomplete resection (vs. complete resection) & $2.19(1.39-3.46)$ & 0.001 & $1.62(0.96-2.66)$ & 0.007 \\
\hline Dexamethasone used (vs. not used) & $2.15(1.34-3.46)$ & 0.002 & $1.36(0.78-2.37)$ & 0.274 \\
\hline Median dose of dexamethasone $\geq 2 \mathrm{mg} /$ day (vs. $<2 \mathrm{mg} /$ day) & $1.90(1.06-3.40)$ & 0.031 & $1.23(0.42-1.64)$ & 0.598 \\
\hline Lymphopenia (vs. no lymphopenia) & $1.99(1.28-3.09)$ & 0.002 & $2.08(1.33 \mathrm{v} 3.25)$ & 0.001 \\
\hline
\end{tabular}

${ }^{*}$ Multivariate analysis was performed on the variables with $p$-values $<0.2$. CCRT, concomitant chemoradiation; ECOG, Eastern Cooperative Oncology Group; CI, confidence interval

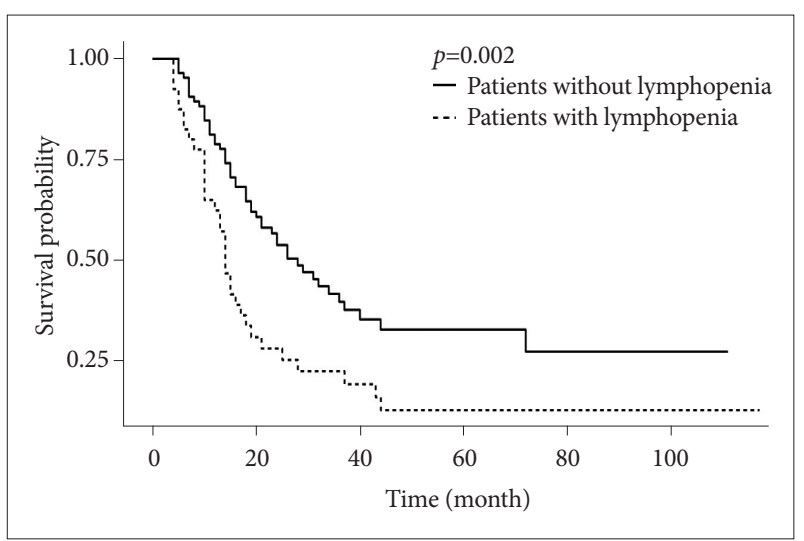

Fig. 1. Kaplan-Meier survival curves of overall survival for patients with lymphopenia vs. without lymphopenia.

\section{Lymphopenia and overall survival}

Kaplan-Meier analysis and multivariate Cox regression analysis with previously identified prognostic factors were performed to evaluate the effect of lymphopenia and higher use of dexamethasone on OS (Table 2). The Kaplan-Meier curves showed that the median OS of patients with lymphopenia was shorter than that of patients without lymphopenia (14.0 months vs. 28.0 months, $p=0.002$ ) (Fig. 1) and the median OS of patients who took dexamethasone was shorter than that of patients who did not take dexamethasone (15.0 months vs. 28.0 months, $p=0.001$ ) (Fig. 2). On multivariate analysis, lymphopenia $(<1,000$ cells/ $\mu \mathrm{L})$ was independently associated with shorter survival (HR 2.08, $p<0.001$ ), while dexamethasone use alone and dexamethasone dose greater than 2 $\mathrm{mg} /$ day were not. Well-known prognostic factors, including non-GTR and an ECOG score of 2 or more, were significantly associated with shorter survival (HR: 1.62, $p=0.007$, and HR: $0.46, p=0.002$ ).

\section{Lymphopenia and risk factors}

Multivariate logistic regression was performed to identify independent clinical factors associated with lymphopenia (Table 3). Female sex and dexamethasone dose greater than 2

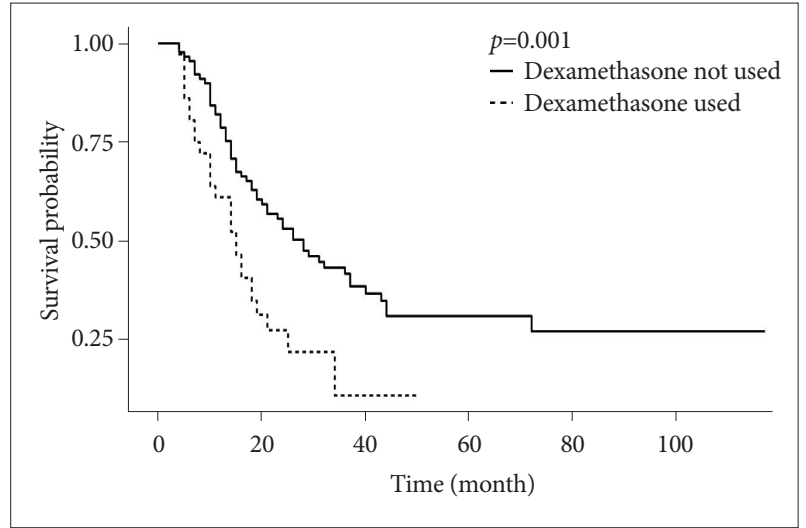

Fig. 2. Kaplan-Meier survival curves of overall survival for patients who took dexamethasone vs. did not take dexamethasone.

mg per day were independently associated with lymphopenia (male vs. female, HR: 0.31, $p=0.003$, and HR: $2.85, p=0.032$, respectively), while use of dexamethasone regardless of dose was not associated with lymphopenia ( $\operatorname{HR} 1.45, p=0.238$ ). Older age, non-GTR, PTV, RT modality, and baseline TLC count were not associated with lymphopenia.

\section{DISCUSSION}

In our study, we showed that lymphopenia was an independent prognostic factor in terms of OS as were already wellknown parameters including EOR and performance status. We also found that female sex and median daily dexamethasone dose greater than $2 \mathrm{mg}$ from initiation of CCRT to 4 weeks after completion of CCRT were independently associated with lymphopenia in patients with newly diagnosed GBM. However, dexamethasone dose less than $2 \mathrm{mg} /$ day, EOR, baseline TLC, RT modality (IMRT vs. 3DRT), and PTV were not significantly associated with lymphopenia. In addition, the use of dexamethasone, regardless of dose, was not independently associated with OS.

Dexamethasone, as a synthetic glucocorticoids, bind to the glucocorticoid receptor, which is expressed in almost all im- 
Table 3. Univariate and multivariate logistic regression for acute lymphopenia (TLC<1,000 cells/ $\mu \mathrm{L}) 4$ weeks after completion of CCRT

\begin{tabular}{|c|c|c|c|c|}
\hline \multirow{2}{*}{ Parameters } & \multicolumn{2}{|c|}{ Univariate analysis } & \multicolumn{2}{|c|}{ Multivariate analysis* } \\
\hline & Hazard ratio $(95 \% \mathrm{CI})$ & $p$-value & Hazard ratio $(95 \% \mathrm{CI})$ & $p$-value \\
\hline Sex (male vs. female) & $0.28(0.12-0.61)$ & 0.002 & $0.31(0.14-0.71)$ & 0.003 \\
\hline Age $\geq 65$ yr (vs. $<65$ yr) & $0.68(0.29-1.58)$ & 0.369 & - & - \\
\hline ECOG 0 or 1 (vs. 2 or 3$)$ & $0.82(0.38-1.76)$ & 0.604 & - & - \\
\hline Incomplete resection (vs. complete resection) & $1.99(0.91-4.38)$ & 0.087 & $1.14(0.43-3.04)$ & 0.798 \\
\hline IMRT (vs. 3DRT) & $0.76(0.32-1.77)$ & 0.520 & - & - \\
\hline PTV $\geq 250 \mathrm{~cm}^{3}$ (vs. $<250 \mathrm{~cm}^{3}$ ) & $1.66(0.78-3.54)$ & 0.190 & $1.70(0.72-4.04)$ & 0.228 \\
\hline Higher baseline TLC $(1,000$ cell $/ \mu \mathrm{L})$ & $0.99(0.94-1.03)$ & 0.566 & - & - \\
\hline Use of dexamethasone (vs. not use) & $2.17(0.97-4.86)$ & 0.060 & $1.45(0.56-3.75)$ & 0.238 \\
\hline Median dose of dexamethasone $\geq 2 \mathrm{mg} /$ day (vs. $<2 \mathrm{mg} /$ day) & $3.21(1.16-8.91)$ & 0.025 & $2.85(0.98-8.28)$ & 0.032 \\
\hline
\end{tabular}

${ }^{*}$ Multivariate analysis was performed using the variables with $p$-values $<0.2$. CCRT, concomitant chemoradiation; ECOG, Eastern Cooperative Oncology Group; IMRT, intensity modulated radiation therapy; 3DRT, three-dimensional radiation therapy; PTV, planning treatment volume; TLC, total lymphocyte count; CI, confidence interval

mune cells [26]. Recent preclinical experiments using conditional glucocorticoid receptor-knockout mice as well as transgenic mice showed effects of glucocorticoid on immune and inflammatory responses [27-29]. In the thymus, glucocorticoid induced apoptosis of naive $\mathrm{T}$ cell and resulted in regulation of $\mathrm{T}$ cell number and function during development $[27,28]$. In the periphery, glucocorticoid also promoted apoptosis of $\mathrm{T}$ cells in lymphoid organs and limited the migration activity of $\mathrm{T}$ cells to the site of inflammation [29].

Huang et al. [11] showed that acute severe lymphopenia was associated with higher brain volume receiving $25 \mathrm{~Gy}$ (V25Gy) [odds ratio (OR): 1.03, CI: 1.003-1.05], female sex (OR: 5.30, CI: 2.46-11.41), older age (OR: 1.05, CI: 1.02-1.09), and lower baseline TLC (OR: 0.92, CI: 0.87-0.98) in patients with high grade glioma who were treated with RT and TMZ chemotherapy. Rudra et al. [14] also found that higher brain volume receiving $25 \mathrm{~Gy}$ (OR: 1.048, CI: 1.022-1.074), older age (OR: 1.091 CI: 1.047-1.137), and female sex (OR: 2.901, CI: 1.391-6.047) were independently associated with acute severe lymphopenia in patients with GBM treated with CCRT. In addition, Lin et al. [15] found that older age (OR: 1.06, CI: 1.02-1.10), female sex (OR: 10.08, CI: 3.79-30.5), and TMZ use (OR: 72.3, CI: 7.8-679) were independently associated with lymphopenia after RT and/or TMZ in patients with high grade glioma. However, these three studies did not find that dexamethasone use was associated with lymphopenia. A recent study published by Hui et al. [17] calculated the cumulative exposure of dexamethasone during CCRT and showed that increased use of dexamethasone was independently associated with acute severe lymphopenia and OS. Chitadze et al. [13] showed that dexamethasone affects various immune cell parameters not including TLC in GBM patients. Based on our findings and previous reports, female sex seemed to be strongly associated with lymphopenia. Further investigation is needed to determine why females showed more frequent lymphopenia than males.

In this study, we calculated the overall cumulative dose of dexamethasone from initiation of CCRT to 4 weeks after completion of CCRT (the day before starting the first round of adjuvant TMZ chemotherapy), which was also the same date blood samples were collected for calculating TLC. Most of the studies mentioned above included only baseline dose of dexamethasone and did not include overall cumulative dose from initiation of CCRT until blood sampling. We found that a median daily dexamethasone dose greater than $2 \mathrm{mg}$ from initiation of CCRT until the start of the first round of adjuvant TMZ chemotherapy was independently associated with lymphopenia, while a dexamethasone dose less than $2 \mathrm{mg}$ per day was not.

Our findings provide evidence that higher cumulative dexamethasone dose during CCRT in patients with newly diagnosed GBM could be a significant risk factor for developing lymphopenia. A large body of evidence from preclinical and clinical studies in other cancers supports the adverse effect of dexamethasone on patient immunity [20,30,31]. However, the use of dexamethasone itself was not independently associated with OS in our study. This may be due to other confounding factors and a larger prospective analysis considering various confounding factors is needed to further evaluate the potential relationship between dexamethasone use and lymphopenia.

Our study had several limitations. There is a possibility of selection bias due to its retrospective and non-randomized design. Molecular parameters, such as MGMT gene methylation, IDH1, and 1p19q co-deletion were not fully identified. More patients in the dexamethasone group underwent nonGTR surgery. Remaining microscopic and/or macroscopic tumor cells could also have induced immunosuppression. Further investigation of the immunosuppressive effect of remnant tumor cells is needed, although a higher dose of dexa- 
methasone was independently associated with acute lymphopenia in our study.

In conclusion, use of dexamethasone itself after initiation of CCRT until 4 weeks after completion of CCRT was not associated with developing lymphopenia at 4 weeks after completion of CCRT. However, the median daily dexamethasone dose $\geq 2 \mathrm{mg}$ was independently associated with lymphopenia. These results should be validated by further prospective studies.

\section{Conflicts of Interest}

The authors have no potential conflicts of interest.

\section{Acknowledgments}

This research was supported by the Bio and Medical Technology Development Program of the National Research Foundation funded by the Ministry of Science and ICT (NRF-2020M3A9E8024875).

\section{ORCID iDs}

Stephen Ahn (D) https://orcid.org/0000-0002-6854-1597
Changik Lee (D) https://orcid.org/0000-0002-6264-8617

\section{REFERENCES}

1. Ray-Coquard I, Cropet C, Van Glabbeke M, et al. Lymphopenia as a prognostic factor for overall survival in advanced carcinomas, sarcomas, and lymphomas. Cancer Res 2009;69:5383-91.

2. Grossman SA, Ye X, Lesser G, et al. Immunosuppression in patients with high-grade gliomas treated with radiation and temozolomide. Clin Cancer Res 2011;17:5473-80.

3. Mendez JS, Govindan A, Leong J, Gao F, Huang J, Campian JL. Association between treatment-related lymphopenia and overall survival in elderly patients with newly diagnosed glioblastoma. J Neurooncol 2016;127:329-35.

4. Campian JL, Piotrowski AF, Ye X, et al. Serial changes in lymphocyte subsets in patients with newly diagnosed high grade astrocytomas treated with standard radiation and temozolomide. J Neurooncol 2017; 135:343-51.

5. Ahn S, Park JS, Jang J, et al. The association between total lymphocyte count after concomitant chemoradiation and overall survival in patients with newly diagnosed glioblastoma. J Clin Neurosci 2020;71:21-5.

6. Kim WJ, Dho YS, Ock CY, et al. Clinical observation of lymphopenia in patients with newly diagnosed glioblastoma. J Neurooncol 2019; 143:321-8.

7. Hanahan D, Weinberg RA. Hallmarks of cancer: the next generation. Cell 2011;144:646-74

8. Restifo NP, Dudley ME, Rosenberg SA. Adoptive immunotherapy for cancer: harnessing the T cell response. Nat Rev Immunol 2012;12: 269-81.

9. Chongsathidkiet $\mathrm{P}$, Jackson C, Koyama S, et al. Sequestration of T cells in bone marrow in the setting of glioblastoma and other intracranial tumors. Nat Med 2018;24:1459-68.

10. Grassberger C, Ellsworth SG, Wilks MQ, Keane FK, Loeffler JS. Assessing the interactions between radiotherapy and antitumour immunity. Nat Rev Clin Oncol 2019;16:729-45.

11. Huang J, DeWees TA, Badiyan SN, et al. Clinical and dosimetric predictors of acute severe lymphopenia during radiation therapy and concurrent temozolomide for high-grade glioma. Int J Radiat Oncol Biol Phys 2015;92:1000-7.

12. Rodríguez DM, Guerrero ME, Maldonado BM, Vollbracht C, Herrera
SA. Total lymphocyte count in cancer patients with lymphopenia treated with intravenous vitamin C: results of an observational study. Transl Med Commu 2017;2:3.

13. Chitadze G, Flüh C, Quabius ES, et al. In-depth immunophenotyping of patients with glioblastoma multiforme: impact of steroid treatment. Oncoimmunology 2017;6:e1358839.

14. Rudra S, Hui C, Rao YJ, et al. Effect of radiation treatment volume reduction on lymphopenia in patients receiving chemoradiotherapy for glioblastoma. Int J Radiat Oncol Biol Phys 2018;101:217-25.

15. Lin AJ, Campian JL, Hui C, et al. Impact of concurrent versus adjuvant chemotherapy on the severity and duration of lymphopenia in glioma patients treated with radiation therapy. J Neurooncol 2018;136:403-11.

16. Ye LL, Fan XW, Hu CS, et al. Dosimetry of the brain and hypothalamus predicting acute lymphopenia and the survival of glioma patients with postoperative radiotherapy. Cancer Med 2019;8:2759-68.

17. Hui CY, Rudra S, Ma S, Campian JL, Huang J. Impact of overall corticosteroid exposure during chemoradiotherapy on lymphopenia and survival of glioblastoma patients. J Neurooncol 2019;143:129-36.

18. Byun HK, Kim N, Yoon HI, et al. Clinical predictors of radiation-induced lymphopenia in patients receiving chemoradiation for glioblastoma: clinical usefulness of intensity-modulated radiotherapy in the immuno-oncology era. Radiat Oncol 2019;14:51.

19. Piotrowski AF, Nirschl TR, Velarde E, et al. Systemic depletion of lymphocytes following focal radiation to the brain in a murine model. Oncoimmunology 2018;7:e1445951.

20. Wong ET, Lok E, Gautam S, Swanson KD. Dexamethasone exerts profound immunologic interference on treatment efficacy for recurrent glioblastoma. Br J Cancer 2015;113:1642.

21. Pitter KL, Tamagno I, Alikhanyan K, et al. Corticosteroids compromise survival in glioblastoma. Brain 2016;139:1458-71.

22. Cenciarini M, Valentino M, Belia S, et al. Dexamethasone in glioblastoma multiforme therapy: mechanisms and controversies. Front Mol Neurosci 2019;12:65.

23. Sampson JH, Maus MV, June CH. Immunotherapy for brain tumors. J Clin Oncol 2017;35:2450-6.

24. Lacroix M, Abi-Said D, Fourney DR, et al. A multivariate analysis of 416 patients with glioblastoma multiforme: prognosis, extent of resection, and survival. J Neurosurg 2001;95:190-8.

25. Ahn S, Park JS, Song JH, Jeun SS, Hong YK. Effect of a time delay for concomitant chemoradiation after surgery for newly diagnosed glioblastoma: a single-institution study with subgroup analysis according to the extent of tumor resection. World Neurosurg 2020;133:e640-5.

26. Coutinho AE, Chapman KE. The anti-inflammatory and immunosuppressive effects of glucocorticoids, recent developments and mechanistic insights. Mol Cell Endocrinol 2011;335:2-13.

27. Purton JF, Monk JA, Liddicoat DR, et al. Expression of the glucocorticoid receptor from the $1 \mathrm{~A}$ promoter correlates with $\mathrm{T}$ lymphocyte sensitivity to glucocorticoid-induced cell death. J Immunol 2004;173: 3816-24.

28. Herold MJ, McPherson KG, Reichardt HM. Glucocorticoids in T cell apoptosis and function. Cell Mol Life Sci 2006;63:60-72.

29. Wüst S, van den Brandt J, Tischner D, et al. Peripheral T cells are the therapeutic targets of glucocorticoids in experimental autoimmune encephalomyelitis. J Immunol 2008;180:8434-43.

30. Gustafson MP, Lin Y, New KC, et al. Systemic immune suppression in glioblastoma: the interplay between CD $14^{+} \mathrm{HLA}-\mathrm{DR}{ }^{\mathrm{lo} / \mathrm{neg}}$ monocytes, tumor factors, and dexamethasone. Neuro Oncol 2010;12:631-44.

31. Shields LB, Shelton BJ, Shearer AJ, et al. Dexamethasone administration during definitive radiation and temozolomide renders a poor prognosis in a retrospective analysis of newly diagnosed glioblastoma patients. Radiat Oncol 2015;10:222. 